\title{
Li-Fi: Velocidad de Internet sorprendente bajo la Luz Visible
}

\section{Li-Fi: Surprising Internet Speed Under Visible Light}

\author{
Moises Espinosa $^{1 *}$ y Marco Vivanco ${ }^{2}$ \\ ${ }^{1}$ Universidad Católica de Cuenca \\ Macas, 140150, Ecuador \\ ${ }^{2}$ Escuela Superior Politécnica de Chimborazo \\ Riobamba, 060150, Ecuador \\ *respinozat@ucacue.edu.ec
}

\begin{abstract}
Resumen
La presente investigación presenta una revisión sobre la trasmisión de datos a través de la tecnología LI-FI, conocido también como el Internet de las cosas (IoT). Para ello se remontará a sus inicios, en los años noventa, donde se inició con el uso de las comunicaciones inalámbricas; dejando de lado las comunicaciones a través de cables para posteriormente buscar la trasmisión de datos a grandes velocidades de manera inalámbrica. De esto se deriva la nueva tecnología que utiliza la luz como medio de trasmisión LI-FI, la misma que a través de LED utiliza los haces de luz para trasmitir grandes cantidades de datos a grandes velocidades que podrían alcanzar los $10 \mathrm{G} / \mathrm{s}$. Esto vendría a remplazar el uso de lo que hasta el momento se ha venido utilizando como medio de trasmisión inalámbrico, el Wifi. LIFI dará solución a las saturaciones y la baja tasa de transferencia de datos en lugares con grandes cantidades de usuarios y dispositivos que pretenden conectarse al acceso a la red a través de WIFI. LIFI viene a remplazar esta tecnología dando solución a los problemas detectados con el uso de WIFI; esta nueva tecnología se ha estudiado desde los años 2010 en la Universidad de Edimburgo, Escocia por el Profesor Ph.D. Harald Haas, y ha sido comercializada en el año 2011. En la actualidad ya muchos países han implementado en sus redes este tipo de tecnología. Sin embargo, esta tecnología se ve dificultada por algunos problemas. En este artículo vamos a repasar brevemente esta tecnología y discutir algunos de estos problemas y sus posibles soluciones.
\end{abstract}

Palabras clave: Internet de las Cosas, Li-Fi, LED, VLC, Wi-Fi.

\begin{abstract}
This research work presents a review on data transferring through LI-FI technology, also known as the Internet of Things (loT). In order to achieve this, it will be necessary to go back to its beginnings in the 1900s, where the use of wireless communications started, leaving aside wired communications, to later on look into high-speed-wireless data transfer. This has given rise to a new technology that uses light as a means of transfer (LI-FI). It uses light beams through LED technology, in order to transfer large amounts of data at speeds as high as $10 \mathrm{G} / \mathrm{s}$. LI-FI would replace the use of what has, thus far, been used as a means of wireless transfer, the WiFi. It will provide solution problems due to overload and low data transfer rates in places with large amounts of devices and users who want to access the network through WIFI. LIFI will replace this technology by solving the problems detected with the use of WIFI; this new technology has been studied since 2010 at the University of Edinburgh, Scotland by Professor PhD. Harald Haas, and it has been marketed since 2011. Many countries have already implemented this type of technology in their networks; however, this technology is hampered by some problems. In this article we will briefly review this technology and discuss some of these problems as well as their possible solutions.
\end{abstract}

Key words: Internet of Things, Li-Fi, LED, VLC, Wi-Fi.

\section{INTRODUCCIÓN}

$\mathrm{E}$ L término LiFi (Light Fidelity) es usado para etiquetar a esta nueva tecnología que se ofrece como alternativa al WiFi, que usa la luz de un diodo emisor de luz LED y es capaz de potenciar velocidades de hasta tres gigabytes por segundo (Gbps). Este sistema de comunicaciones inalámbrico es rápido, de bajo costo y de una mayor velocidad de transmisión.

Las contribuciones de este trabajo están enfocadas en el análisis de la tecnología Li-Fi relacionada con el Internet de las cosas que permitirá tener infinidad de objetos conectados a Internet y aprovechar el espectro de luz que emiten las bombillas LED lograr transmitir audio y video a grandes velocidades.

Este trabajo se encuentra organizado en seis secciones. La Sección 1 hace referencia a la parte Introductoria. La Sección 2 se enfoca en el estado del arte de los temas investigados divididos en subsecciones como son: la subsección A representa un análisis de la problemática que presenta la tecnología Wi-Fi, el uso más frecuente 
de esta tecnología es la conexión de portátiles a Internet desde las cercanías de un punto de acceso o hotspot, el principal problema radica en la progresiva saturación del espectro radioeléctrico y su elevado porcentaje de redes que son instalados sin tener en consideración la seguridad. La subsección B se analiza la Visible Light Communication (VLC) un medio para transmitir información, su principal ventaja es la facilidad y comodidad de disponer de Internet con sólo tener la luz en casa, su limitante e que sólo es posible disponer de gran ancho de banda a poca distancia o cobertura de la luz. La subsección $\mathrm{C}$ menciona acerca de la nueva tecnología pionera en comunicación inalámbrica de Internet mediante la luz como lo es $\mathrm{Li}-\mathrm{Fi}$, presenta la ventaja de no causar interferencias con otros sistemas y puede utilizarse en áreas sensibles como el interior de un avión. La subsección D representa un análisis de un término ambiguo, pero se está volviendo rápidamente una tecnología tangible como lo es Internet Of The Things es esencialmente un sistema de máquinas u objetos equipados con tecnologías de recopilación de datos, de manera que esos objetos pueden comunicarse entre sí. En la Sección 3 se realiza una comparación de tecnologías inalámbricas como Wi-Fi y Li-Fi. La estructura de la tecnología LiFi sus componentes y el diagrama de bloques se discuten en la sección 4. En la sección 5 se enfocan las diferentes aplicaciones donde se puede utilizar esta tecnología de comunicación. Finalmente esta la Sección 6 tiene que ver con las conclusiones obtenidas de la investigación.

\section{DesarRollo}

El desarrollo de contenidos presenta el marco teórico de cuatro secciones como la problemática de la tecnología WiFi, la comunicación de la luz visible, la tecnología Li-Fi y el enfoque del Internet de las cosas.

\section{A. Problemática Wi-Fi}

Las redes inalámbricas constituyen hoy en día una de las tecnologías con mayor crecimiento tecnológico, ya que ha llegado a tener como características principales una movilidad y una instalación más sencilla, además permite la fácil ampliación de una red. Es decir, que podemos estar en movimiento por cualquier lugar sin perder la conectividad con Internet. Esto es algo que actualmente está tomando gran importancia, ya que con la evolución de las tecnologías el uso de Internet se ha multiplicado, lo que hace posible poder disponer de él en cualquier parte sin "ataduras" de cables.

La tecnología Wi-Fi es un mecanismo de conexión de dispositivos electrónicos de forma inalámbrica. Los dispositivos habilitados con Wi-Fi, tales como un computador, un teléfono celular o un reproductor de audio o vídeo digital, pueden conectarse a Internet a través de un punto de acceso de red inalámbrica. Por la gran cantidad de equipos que utilizan esta tecnología cada vez se vuelve problemática y ocasiona conexiones lentas e inseguras.
Uno de los problemas más graves a los cuales se enfrenta actualmente la tecnología Wi-Fi, es la progresiva saturación del espectro radioeléctrico, debido a la masificación de usuarios, esto afecta especialmente en las conexiones de larga distancia (mayor de 100 metros). En realidad WiFi está diseñado para conectar ordenadores a la red a distancias reducidas, cualquier uso de mayor alcance está expuesto a un excesivo riesgo de interferencias.

Otro problema es el elevado porcentaje de redes que son instaladas sin tener en consideración la seguridad, convirtiendo así sus redes en redes abiertas (o completamente vulnerables a los crackers), sin proteger la información que por ellas circulan. Esta tecnología además de la velocidad limitada de Internet que permite, la parte de seguridad es relativamente flexible y sus costos de implementación son elevados, son algunos de los problemas de Wi-Fi, sin embargo la parte de interferencia de objetos es elevada porque la tecnología Wi-Fi permite travesar paredes y transmitir información de diferentes lugares, basta con no perder la señal de comunicación del router inalámbrico.

\section{B. Comunicación luz visible}

Visible Light Communication ha atraído mucho interés de muchos investigadores y desarrolladores se centran en gran medida en el diseño o el desarrollo de un sistema que proporciona un vínculo excelente para comunicaciones punto a punto [1][2]. La comunicación por luz es visible (a diferencia con la comunicación por radio invisible), por lo que es fácil de determinar quién puede escuchar (o recibir) un mensaje. Por otra parte, la comunicación por luz no utiliza ondas electromagnéticas, y hay entornos o comunidades que puedan valorar este aspecto. Un efecto secundario es que la comunicación por luz no requiere parte de espectro de radio y por lo tanto puede ser utilizada en escenarios de ancho de banda limitado. La trasmisión de datos mediante el uso de luz visible sería mucho más óptima, debido a que está presente en muchos lugares, por lo que existe la posibilidad de combinar la comunicación de luz con el diseño de iluminación para permitir la Comunicación Luz Visible [3][4].

VLC se refiere a la tecnología de comunicación que utiliza la fuente de luz visible como un transmisor de señales, el aire como medio de transmisión, y el fotodiodo adecuado como un componente de la señal de recepción. En la Fig. 1 se observa las bombillas en la línea de visión se comunican entre sí utilizando la óptica de espacio libre.

La luz es substancialmente más segura y puede ser utilizada en lugares donde la comunicación de frecuencia de radio a menudo se considera problemática, por ejemplo en cabinas de los aviones u hospitales. Así VLC no solo tiene el potencial de resolver el problema de falta de espacio de espectro. El espectro de la luz visible no se utiliza, no está regulada, y puede ser usada para comunicaciones a altas velocidades. 


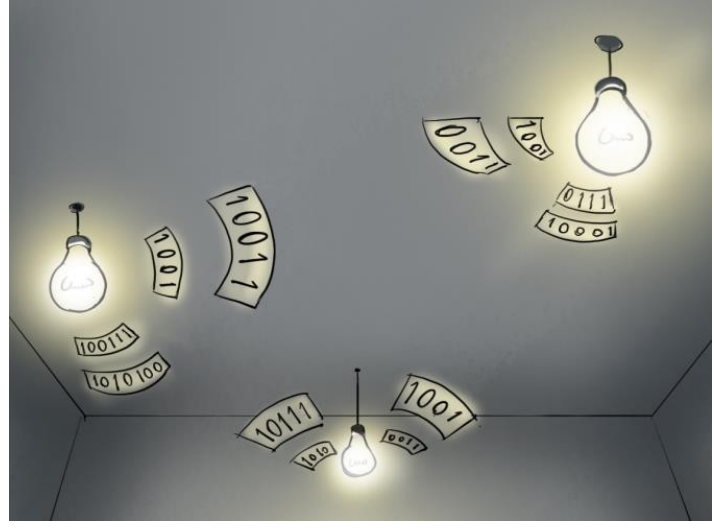

FIG. 1. VLC con bombillas inteligentes.

\section{Tecnología $\mathrm{Li}-\mathrm{Fi}$}

Es común encontrar problemas de interferencia con la señal Wi-Fi por presentarse un exponencial incremento de dispositivos con capacidades inalámbricas y un aumento de la demanda de capacidad de transmisión, cada vez es complicado encontrar canales de radios libres de saturación para nuestras redes inalámbricas, por tal razón se plantea una solución a través de comunicaciones inalámbricas con lámparas basadas en LED para transmitir datos, una nueva tecnología llamada Li- Fi (Light Fidelity).

La idea del sistema surgió hace tres años, "cuando un grupo de investigadores y empresarios mexicanos, encabezados por Arturo Campos, director general de Sisoft México, lograron transmitir audio y video a gran velocidad través del espectro de luz que emiten los focos LED, que parpadean millones de veces por segundo a una velocidad imperceptible a la vista" [1]. LiFi tiene la ventaja de no causar interferencias con otros sistemas y puede utilizarse en áreas sensibles como el interior de un avión. Sin embargo, las ondas de luz usadas no pueden penetrar las paredes.

Básicamente en que consiste Li-Fi en utilizar focos o lámparas LED que emitan luz en el espectro visible para tener una comunicación inalámbrica bidireccional en red, distinta a la transmisión tradicional de Wi-Fi basada en radiofrecuencia, sin necesidad de utilizar el despliegues de redes Wi-Fi o el cableado de datos en los diferentes lugares donde se requieran la conexión de Internet. Mientras que la tecnología Li-Fi permite instalar un transceptor en una computadora portátil o dispositivo móvil y ubicarlo dentro del campo de cobertura de la lámpara que hace veces de punto de acceso para disponer de Internet a velocidades idóneas. En la Fig.2 se muestra una comunicación mediante Li-Fi utilizando lámparas LED como puntos de acceso que envían Internet inalámbricamente a varios dispositivos (computador, PDAs, impresoras) [5].

\section{Internet de las Cosas}

El término Internet de las Cosas o como lo llaman en inglés el Internet Of The Things (IoT), literalmente,

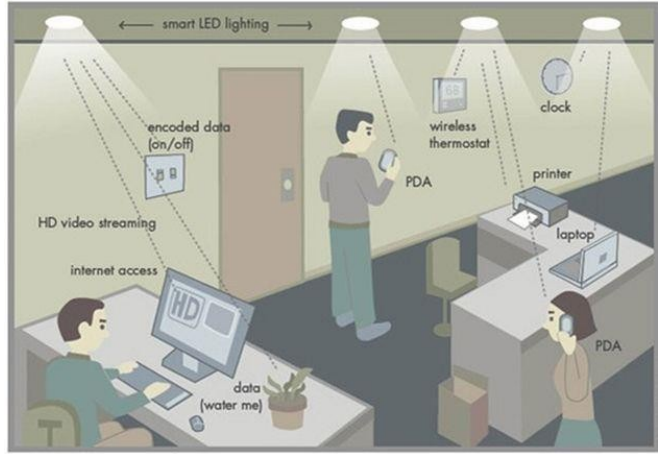

FIG. 2. Comunicación Li-Fi con lámparas LED de punto de acceso a red.

consiste en que todas las cosas tengan conexión a Internet en cualquier momento y lugar. Todo esto se da mediante la integración de sensores y dispositivos en objetos cotidianos que quedan de esta manera conectados a Internet a través de redes fijas e inalámbricas.

IoT puede definirse como una "infraestructura de red global dinámica con capacidad de auto configuración basada en protocolos de comunicación estándar e interoperables donde objetos físicos y virtuales poseen identidades, atributos físicos y personalidades virtuales, utilizan interfaces inteligentes y están perfectamente integrados en la red de información" [6].

Internet ha sido y sigue siendo una revolución y su llegada a dispositivos de uso cotidiano IoT va a generar grandes cambios y, si cabe, generar más necesidad de disponer de estos tipos de dispositivos y, en consecuencia, de mayor conectividad a Internet. Según Cisco Internet Business Solutions Group (IBSG), Internet de las Cosas surgió entre 2008 y 2009 como un simple momento en el tiempo en el que eran más las cosas conectadas a Internet que las personas [7]. En la Fig.3 se muestra un estudio de IBSG de Cisco, en donde se evidencia que la cantidad de dispositivos conectados a Internet sobrepasa significativamente a la población, a una media de 6,58 dispositivos conectados por persona.

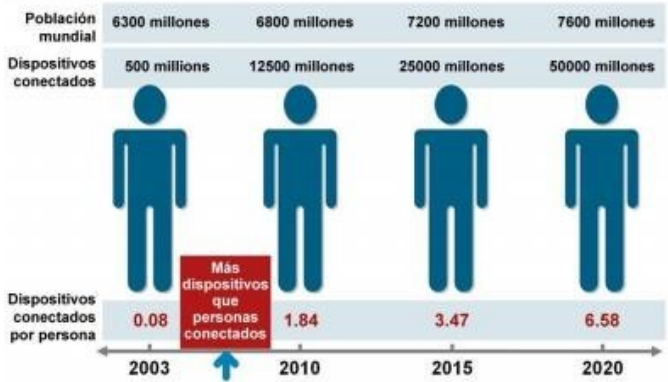

FIG. 3. Índice de dispositivos conectados según estudio de CISCO.

Con el Internet de las cosas, los millones de personas y la infinidad de objetos conectados al Internet ocasionan un planeta interconectado, al tiempo que se vuelve más 
inteligente. Su objetivo principal de IoT es la creación de entornos inteligentes y cosas conscientes para aplicaciones relacionadas con el clima, la alimentación, la energía, la movilidad, la sociedad digital y la salud. El futuro del Internet es prometedor mediante la integración del IoT y las diferentes tecnológicas de comunicación del Internet como WiFi, LiFi, Wimax, etc., tendremos una interconexión con todos los entornos del mundo real [8] como se las detalla a continuación:

- Ciudades (Smart Cities)
- Estacionamientos Inteligentes
- Gestión de Residuos
- Iluminación Inteligente
- Mapas de Ruido Urbano

- Medioambiente Inteligente

- Detección de incendios forestales

- Contaminación del aire

- Detección temprana de terremotos

- Transporte Inteligente

- Sistema de transporte inteligente

- Control automatizado de señales de tránsito

- Edificios Inteligentes

- Energía Inteligente

- Smart Grid: control y gestión del consumo de energía.

- Instalaciones fotovoltaicas

- Salud Inteligente

- Detección de caídas

- Cuidado de deportistas

- Vigilancia de pacientes

- Radiación Ultravioleta

En la Fig.4 se muestra un entorno de Internet de las cosas y la creación de los diferentes entornos inteligentes que a futuro la mayoría de objetos tendrán algún tipo de conectividad inalámbrica

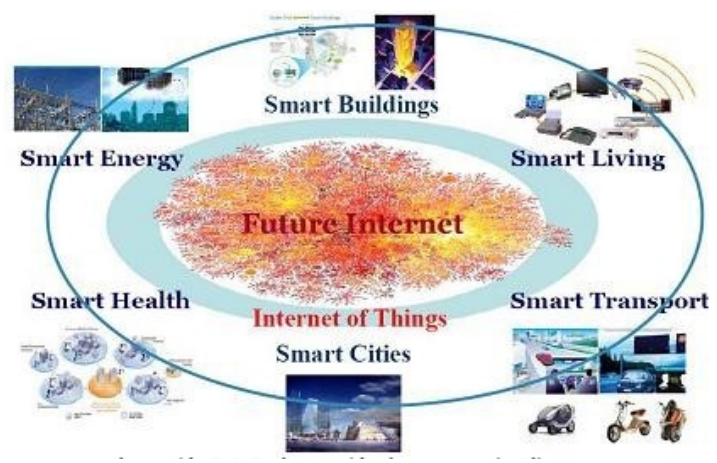

FIG. 4. IoT y la creación de entornos inteligentes

\section{COMPARATIVA ENTRE TECNOLOGÍAS WI-FI Y LI-FI}

Aunque la tecnología Li-Fi aún está en fase de experimentación cabe destacar que promete ser la red inalám- brica del futuro cercano y que sustituirá con el tiempo al conocido sistema inalámbrico Wi-Fi. A continuación la tabla I muestra la comparación entre Wi-Fi y Li-Fi [9] en donde se evalúan distintos parámetros importantes de ambas tecnologías de comunicación inalámbrica de Internet

TABLA I

COMPARATIVA ENTRE LAS TECNOLOGÍAS INALÁMBRICAS WI-FI Y LI-FI

\begin{tabular}{|c|c|c|c|}
\hline \multirow{2}{*}{ \# } & \multirow{2}{*}{ PARAMETROS } & \multicolumn{2}{|c|}{ TECNOLOGIAS INALAMBRICAS } \\
\hline & & Wi-Fi & Li-Fi \\
\hline 1 & Estándar & IEEE 802.11 & IEEE 802.15 \\
\hline 2 & $\begin{array}{l}\text { Velocidad para transmisión de } \\
\text { datos }\end{array}$ & $\begin{array}{l}\text { Minima velocidad } 150 \mathrm{Mb} / \mathrm{s} \\
\text { Máxima velocidad } 1 \mathrm{~Gb} / \mathrm{s}\end{array}$ & $\begin{array}{l}\text { Minima velocidad } 1 \mathrm{~Gb} / \mathrm{s} \\
\text { Máxima velocidad } 10 \mathrm{Gg} / \mathrm{s}\end{array}$ \\
\hline 3 & $\begin{array}{l}\text { Medios de transferencia de } \\
\text { datos }\end{array}$ & Utiliza ondas de radio & Utiliza ondas de luz \\
\hline 4 & Rango de Espectro & $\begin{array}{l}\text { E1 rango del espectro de radio } \\
\text { frecuencias es menos de espectro de } \\
\text { luz visible. }\end{array}$ & $\begin{array}{l}\text { El espectro de la luz visible tiene } \\
\text { amplio espectro } 100.000 \text { tiempo en } \\
\text { comparación con radiofrecuencia }\end{array}$ \\
\hline 5 & Costo & $\begin{array}{l}\text { Caro en comparación con Li-Fi } \\
\text { porque utiliza el espectro } \\
\text { radioeléctrico }\end{array}$ & $\begin{array}{l}\text { Es más barato que } W_{i} \text {-Fi, se dice que } \\
\text { hasta } 10 \text { veces más barato }\end{array}$ \\
\hline 6 & Topologia de Red & Punto a Punto & Punto a Punto \\
\hline 7 & Frecuencia de Operación & $2.4 \mathrm{Ghz}$ & Cientos de Tera $\mathrm{Hz}$ \\
\hline 8 & Interferencia de objetos & $\begin{array}{l}\text { La radiofrecuencia atraviesa } \\
\text { paredes no se pierde la } \\
\text { comunicación }\end{array}$ & $\begin{array}{l}\text { La luz no atraviesa paredes, si se } \\
\text { obstruye o paga la luz LED se pierde } \\
\text { la comunicación }\end{array}$ \\
\hline 9 & Seguridad & Media seguridad por & $\begin{array}{l}\text { La luz, al no atravesar paredes, es } \\
\text { mucho más segura que el Wi-Fi. }\end{array}$ \\
\hline 10 & Disponibilidad & $\begin{array}{l}\text { Baja disponibilidad en lugares que } \\
\text { no se permite la radiofrecuencia } \\
\text { (aviones, hospitales, bajo el agua) }\end{array}$ & $\begin{array}{l}\text { Alta disponibilidad para llegar a } \\
\text { cualesquier lugar con presencia de } \\
\text { luz. }\end{array}$ \\
\hline 11 & Implementación & $\begin{array}{l}\text { Utiliza Access point para enviar } \\
\text { internet inalámbrico }\end{array}$ & Utiliza bombillas de luz LED \\
\hline 12 & Cableado & $\begin{array}{l}\text { Requiere de cables para llegar a los } \\
\text { diferentes Access point }\end{array}$ & Ausencia de cables \\
\hline
\end{tabular}

\section{ESTRUCTURA DE LI-FI}

Li-Fi es una alta velocidad y la versión óptica económica de Wi-Fi. Se basa en la Comunicación de Luz Visible (VLC). Los principales componentes del sistema de Li-Fi son los siguientes:

a) Un LED blanco de alto brillo, que actúa como la transmisión fuente.

b) Un fotodiodo de silicio con buena respuesta a la luz visible como elemento receptor.

LED se pueden encender y apagar para generar cadenas digitales de disposición diferente de 1 y 0 . Para producir un nuevo flujo de información, los datos pueden ser programados a la luz cambiando la velocidad con gas del LED.

Los LEDs pueden ser usados como un distribuidor, con la ayuda de la modulación de la luz LED de la señal de datos. La productividad LED aparece estable para el ojo humano por activo de la tasa de parpadeo rápido del LED. El sistema emisor Li-Fi consta de 4 subconjuntos principales [10]:

1) Bombilla

2) RF Circuito amplificador de potencia (PA)

3) Placa de circuito impreso (PCB)

4) Caja 
Una nueva generación de diodos emisores de luz de alto brillo constituye la parte fundamental de la tecnología de fidelidad de luz. La lógica es muy simple. Si el LED se enciende, un pulso digital 1 se transmite. Si el LED está desvío, un pulso digital de 0 se transmite. Estos LEDs de alto brillo se pueden encender y apagar rápidamente lo que nos da unas muy buenas oportunidades para la transmisión de datos a través de la luz [11]. El trabajo de Li-Fi es muy simple. Por lo tanto la luz emite en un extremo, por ejemplo, un LED, y un fotodetector (sensor de luz) en el otro extremo. El detector de foto registra un 1 binario cuando el LED está encendido y un 0 binario si el LED está apagado.

El diagrama de bloques de sistema de Li-Fi se muestra en la Fig. 5. Los datos se pueden codificar a la luz mediante la variación de la tasa de parpadeo en el que los LED parpadean y se apagan para generar secuencia diferente de $1 \mathrm{~s}$ y 0s. La intensidad del LED es modulada tan rápidamente que el ojo humano no puede notar que, para que la luz del LED aparece constante para los seres humanos.

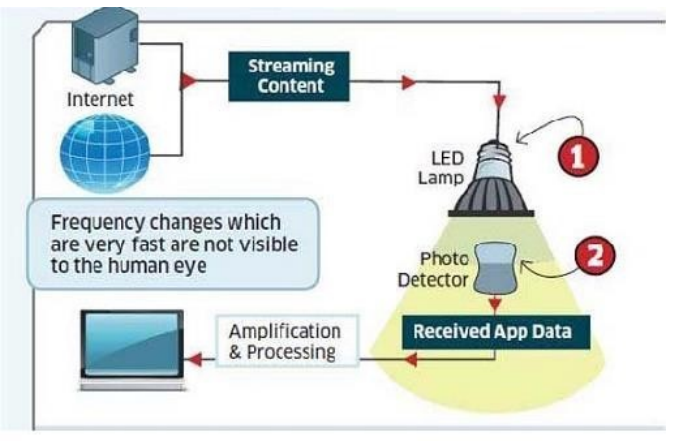

FIG. 5. Diagrama de bloques del Sistema de Li-Fi

\section{APLICABILIDAD CON LI-FI}

Esta tecnología Li-Fi se puede utilizar con el Internet de las Cosas (IoT). Se refiere a la forma única de objetos identificables y sus representaciones virtuales en una estructura como el Internet.

Li-Fi es una fuente de luz de alta intensidad de diseño revolucionario. Esta tecnología no se ocupa de las ondas de radio, por lo que puede ser fácilmente ser utilizado en el lugar donde, Bluetooth, infrarrojos, Wi-Fi no se puede utilizar. A continuación se describen los diferentes entornos donde se puede aplicar la tecnología Li-Fi [12] como:

- Entornos peligrosos: Li-Fi ofrece una alternativa segura a la interferencia electromagnética de comunicaciones de radiofrecuencia en entornos tales como minas, plantas petroquímicas, centrales nucleares, etc.

- El Hospital y Salud: Li-Fi no emite ninguna interferencia electromagnética y así no interferir con los instrumentos médicos, ni es interferido por escáneres de resonancia magnética. Esto incluso puede ser útil para cirugías robóticas, así como otros procedimientos automáticos.
- El Tráfico sin problemas y Transporte: Uso de la luz en el tráfico es fácil recopilar datos de tráfico LED y en consecuencia pueden suavizar, coche incrustado con lámpara LED se pueden comunicar entre sí, debido a las posibilidades de colisión se reduce al mínimo.

- Comunicaciones Subacuáticos: Ondas de radio se absorbe fuertemente en el agua, así que usar esto es poco práctico. Las ondas acústicas tienen muy poco ancho de banda y perturban la vida marina. Li-Fi ofrece una solución para comunicaciones de corto alcance bajo el agua.

- Los sistemas educativos: Li-Fi es la última tecnología que puede proporcionar un acceso más rápido a Internet de velocidad. Por lo tanto, puede reemplazar Wi-Fi gratuita en las instituciones educativas y en las empresas para que todas las personas pueden hacer uso de Li-Fi con la misma velocidad prevista en un área en particular.

- Incrementar la Seguridad en las calles: Se podría utilizar Li-Fi para transmitir datos de video cámaras colocadas en las calles hacia los servicios de emergencia y policía.

- Gestión de desastres: Li-Fi se puede utilizar como un poderoso medio de comunicación en tiempos de desastres tales como terremotos o huracanes. Las personas promedio pueden no conocer los protocolos durante este tipo de desastres. Las estaciones de metro y túneles, zonas muertas comunes para la mayoría de las comunicaciones de emergencia, no representan un obstáculo para el Li-Fi.

\section{CONClusiones}

- La tecnología Li-Fi convertirá el mundo de las comunicaciones en algo accesible para todas las personas, por su bajo costo y fácil de implementar; además es mucho más veloz, seguro y eficiente en comparación con los otros tipos de acceso a Internet que conocemos en la actualidad.

- La compatibilidad de Li-Fi con el Internet de las cosas permitirá tener variedad de aplicaciones conectadas a Internet en diferentes campos lo cual genera una buena rentabilidad, y abrirá puertas a nuevas áreas que no han sido exploradas por falta de recursos.

- Li-Fi tiene una oportunidad brillante para sustituir la tradicional Wi-Fi, su población que utiliza el Internet inalámbrico cada vez es mayor, las ondas se están convirtiendo cada vez más obstruidas, por lo que cada vez es más difícil obtener una señal fiable y de alta velocidad. Li-Fi promete resolver cuestiones como la escasez de ancho de banda de las radiofrecuencias y arrancar las desventajas de Wi-Fi.

- Esta tecnología presenta muchas limitantes que habrá que resolver, por ejemplo la línea de vista, es decir la luz no puede atravesar objetos opacos, mientras las comunicaciones de radiofrecuencia si lo permiten. Es decir, si algo o alguien interfiere la luz emitida por un 
LED, la conexión será interrumpida. Aun no existen protocolos de comunicación que eviten este problema.

- En un futuro cerca las conexiones a Internet mediantes redes inalámbricas serán mejores, rápidas y muy seguras; porque se implementaran protocolos y estándares nuevos para mejorar las comunicaciones.

- El Internet de las cosas, las tecnologías Li-Fi y Wimax serán un mecanismo que ocasionaran una interconexión de redes con todos los entornos y están perfectamente integrados en la red de información.

\section{REFERENCIAS}

[1] T. Komine and M. Nakagawa, "Fundamental analysis for visible-light communication system using led lights," Consumer Electronics, IEEE Transactions on, vol. 50, no. 1, pp. 100-107, 2004.

[2] K. Bandara and Y.-H. Chung, "Reduced training sequence using rls adaptive algorithm with decision feedback equalizer in indoor visible light wireless communication channel," in ICT Convergence (ICTC), 2012 International Conference on, pp. 149-154, IEEE, 2012.

[3] G. Corbellini, S. Schmid, S. Mangold, T. R. Gross, and A. Mkrtchyan, "Demo: Led-to-led visible light communication for mobile applications," in 3rd IEEE Workshop on Optical Wireless Communications (OWC'12), 2012.

[4] N. O. Tippenhauer, D. Giustiniano, and S. Mangold, "Toys communicating with leds: Enabling toy cars interaction," in Consumer Communications and Networking Conference (CCNC), 2012 IEEE, pp. 48-49, IEEE, 2012.

[5] S. Schmid, G. Corbellini, S. Mangold, and T. R. Gross, "Led-to-led visible light communication networks," in Proceedings of the fourteenth ACM international symposium on Mobile ad hoc networking and computing, pp. 1-10, ACM, 2013.

[6] A. d. M. Cluster ICT, "Internet de las cosas: Objetos interconectados y dispositivos inteligentes". Disponible en https://actualidad.madridnetwork.org/imgArticulos/ Documentos/635294387380363206.pdf, 2013. Acceso: 17 Nov. 2015.
[7] J. O. Navas, "Un acercamiento al estado del arte en cloud computing", Vínculos, vol. 10, no. 2, pp. 157$172,2014$.

[8] A. W. Burange and H. D. Misalkar, "Review of internet of things in development of smart cities with data management \& privacy," in Computer Engineering and Applications (ICACEA), 2015 International Conference on Advances in, pp. 189-195, IEEE, 2015.

[9] B. Ghosal and A. K. Panda, "Li-fi a green energy initiative," International Journal of Computer Applications, vol. 95, no. 11, 2014.

[10] S. V. Kumar, K. Sudhakar, and L. S. Rani, "Emerging technology li-fi over wi-fi," International Journal of Inventive Engineering and Sciences (IJIES), vol. 2, no. 3, 2014.

[11] J. Condliffe, "Will li-fi be the new wi-fi?," New Scientist, vol. 28, 2011.

[12] G. K. PATIDAR, "Li-fi technology in wireless communication,” 2014.

Recibido: 19 de febrero de 2017

Aceptado: 20 de mayo de 2017

Moisés Espinosa: Su formación de Tercer Nivel en el área de Programación de Sistemas, formación de Cuarto Nivel es en Docencia Universitaria y Gestión Educativa, cursando Maestría en Redes e Interconectividad.

Marco Vivanco: Graduado en la Escuela de Informática de la Universidad Técnica Particular de Loja, Ecuador. Analista de Soporte Técnico Provincial en la Coordinación Zonal 7 del Registro Civil y maestrante en la Escuela Superior Politécnica del Chimborazo en la Maestría de Interconectividad de Redes. Correo electrónico: mvvivancog@gmail.com 